Preface

\title{
Controversies in Inherited Bleeding Disorders
}

\author{
Antonio Coppola, MD ${ }^{1}$ Annarita Tagliaferri, MD ${ }^{2}$ Massimo Franchini, MD ${ }^{3}$ \\ 1 Regional Reference Center for Coagulation Disorders, Federico II \\ University Hospital Naples, Italy \\ 2 Regional Reference Center for Inherited Bleeding Disorders, \\ University Hospital of Parma, Italy \\ 3 Department of Transfusion Medicine and Hematology, Carlo Poma \\ Hospital, Mantova, Italy \\ Semin Thromb Hemost 2016;42:459-462.
}

Recent years are witnessing key developments in treatment approaches for congenital bleeding disorders (CBD), in particular the hemophilias, but also other factor deficiencies. ${ }^{1,2}$ Moreover, cooperative multinational efforts are providing advances in our knowledge of pathophysiological, clinical, and management aspects of these disorders, including the more rare abnormalities. ${ }^{3-6}$ However, despite being in an era of novel insights and paradigms of treatment, many issues remain controversial or need further research and/or confirmation in clinical practice. Along these lines, the 16 chapters presented in this latest bumper issue of Seminars in Thrombosis and Hemostasis deal with some of these debated or evolving concepts, presenting and discussing the most recent literature information and the available expert recommendations.

The assessment of patients with suspected or known CBD is based on accurate and appropriate data from clinical history and laboratory testing. Therefore, two papers at the beginning of the issue focus on these crucial aspects. ${ }^{7,8}$ Starting from the clinical validation in type 1 von Willebrand disease (VWD), Tosetto reports on the potential clinical usefulness of bleeding assessment tools (BAT), these being first developed essentially as research tools for the quantification of bleeding symptoms and the study of phenotype/ genotype correlations. ${ }^{7}$ Indeed, although disease-specific implementations should be widely validated in most cases, these tools can provide a main advantage of standardizing the diagnostic process, allowing a rational approach to the laboratory diagnosis, in particular for mild bleeding disorders that may require a complex laboratory work-up. Moreover bleeding severity assessed by these tools has been shown to correlate with the long-term probability of bleeding, thus representing an interesting predictor of disease severity. ${ }^{7}$ As regards laboratory issues, in view of the general lack of agreed approaches for the diagnostics of bleeding disorders, Lippi and colleagues provide pragmatic guidance to improve the

Address for correspondence Antonio Coppola, MD, Regional Reference Center for Coagulation Disorders, Federico II University Hospital, Via S. Pansini 5, 80131, Naples, Italy (e-mail: antocopp@unina.it). identification and diagnosis of CBD due to abnormalities of secondary hemostasis, even in nonspecialized laboratories. ${ }^{8}$ A strategy based on the collection of personal and family history and the results of first-line tests (i.e., prothrombin time, activated partial thromboplastin time, and fibrinogen) is proposed, followed by second- or third-line tests that will definitely establish the specific nature and the severity of the bleeding defect. Indeed, second-line tests (specific factor assays, on the basis of the detected abnormalities in firstline assays) will provide the basis for a preliminary diagnosis, which will then be supported by third-line tests (namely, immunological assays of clotting factors and molecular biology). ${ }^{8}$

VWD is the most common CBD; however, its identification and management still provides many challenges. ${ }^{9}$ Three articles in this issue deal with these problems, reflecting a complex and not completely elucidated pathophysiological background. Castaman and Federici review recent evidence regarding one of the most intriguing VWD subtypes, type $2 \mathrm{~B} .{ }^{10}$ This rare autosomally dominant inherited variant is due to mutations clustered in exon 28 of the von Willebrand Factor (VWF) gene encoding for the A1 domain, involved in VWF binding to platelet glycoprotein Ib- $\alpha$. The mutant VWF, normally synthesized and assembled by endothelial cells, shows heightened affinity binding to its platelet receptor. This results in in vivo platelet clumping that is correlated with a variable degree of thrombocytopenia, in particular under specific clinical circumstances. However, recent studies show that a true platelet defect is also present in VWD 2B, since morphological and functional defects are detected in these patients, possibly contributing to the clinical and laboratory heterogeneity. ${ }^{10}$ In the frame of the phenotypical variability in type 2 VWD, the often misdiagnosed subtypes $2 \mathrm{~A}$ and $2 \mathrm{M}$ are being increasingly studied, showing both similarities and differences in various aspects of their presentation to clinicians, researchers, and laboratories. Favaloro and colleagues
Copyright (C) 2016 by Thieme Medical Publishers, Inc., 333 Seventh Avenue, New York, NY 10001, USA. Tel: +1(212) 584-4662. 10.1055/s-0036-1581101. ISSN 0094-6176.
DOI http://dx.doi.org/
Inherited Bleeding Disorders;

Guest Editors: Antonio Coppola, MD, Massimo Franchini, MD, and Annarita Tagliaferri, MD. 
address, in a comprehensive review, the available data regarding prevalence, genetic defects, laboratory test results, clinical features, and treatment responses of these two VWD subtypes, focusing on differences that gain relevance in the perspective of personalized approaches to management of VWD patients. ${ }^{11}$ Among these strategies, recent reports highlight the role of long-term prophylaxis with $\mathrm{VWF} /$ Factor VIII (FVIII) concentrates in patients with more severe disease, particularly those with type $3 \mathrm{VWD}$, who experience recurrent joint or mucosal bleeds, including epistaxis, menorrhagia, and gastrointestinal bleeding. Reviewing the available studies, Saccullo and Makris show how, despite some uncertainties about the long-term outcomes and economic impact, prophylaxis with VWF-containing concentrates should now be considered as the standard of care in VWD patients with severe bleeding phenotype. ${ }^{12}$

Mild hemophilia (MH) is another challenging bleeding setting, mostly because of delayed diagnosis and unclear therapeutic approach. Desmopressin is an effective, safe, and cheap treatment for patients with $\mathrm{MH} \mathrm{A}$; however, a significant proportion of them do not attain sufficient hemostatic postinfusion FVIII levels, in particular for major surgery. In these cases, replacement therapy with FVIII concentrates is needed. Recent data indicate that MH A patients may develop inhibitors against FVIII, with a lifelong risk, in contrast to severe or moderate hemophilia A. Specific F8 missense mutations, as well intensive treatment, predispose to inhibitor development. ${ }^{5}$ These novel data, together with other emerging issues in the diagnosis and biology of $\mathrm{MH} \mathrm{A}$, are reviewed by Castaman and coworkers. ${ }^{13}$ They also summarize the management of $\mathrm{MH} \mathrm{A}$ inhibitor patients, in which bleeding is treated with desmopressin, high doses of FVIII concentrate or bypassing agents, and inhibitor eradication is variably managed (watchful waiting, immunosuppression, or immune tolerance induction regimens). ${ }^{13}$

"We stand on the threshold of a new era in hemophilia therapy," Giangrande writes at the beginning of his paper in this issue, referring to modified recombinant FVIII and factor IX (FIX) concentrates with extended half-life (EHL), already licensed in some countries, and to clinical trials of gene therapy in both hemophilia A and B. ${ }^{14}$ This and two further contributions in this issue deal with these and additional attractive perspectives in treatment of hemophilia. ${ }^{14-16}$ Giangrande critically reviews the safety and efficacy of EHL concentrates and gene therapy, comparing the potential benefits and disadvantages of these two therapeutic options. EHL concentrates can reduce the burden of intravenous injections, particularly in hemophilia B, and allow higher factor trough levels, with personalization of treatment according to the patient lifestyle. However, preserving patient adherence, the potential prolonged exposure to low factor levels, inhibitor development in previously untreated patients (PUPs), and the actual implementation related to the economic affordability of these products are still poorly known. As regards gene therapy, which offers the prospect of a definitive cure, the feasible timeline remains unclear, in spite of recent encouraging results, particularly in hemophilia $B$ with a sustained FIX level of $0.05 \mathrm{IU} / \mathrm{ml}$ maintained for over
4 years. ${ }^{17}$ A major barrier of gene therapy is the high seroprevalence of antibodies to adeno-associated virus $(A A V)$ vectors in the general population. Moreover, perplexities arise from recent data in other settings indicating that gene therapy is unlikely to be a cheap therapeutic option. Furthermore, the factor levels achieved, although above the current usual target trough levels, fall well below those achievable by prophylaxis with EHL products and considered safe to enable an active lifestyle. ${ }^{14}$ Berntorp and GretenkortAndersson report thoroughly the characteristics of the four EHL FVIII and three FIX products already available or in advanced clinical development, which show a half-life extension of approximately 1.5-fold for FVIII and 2.5-fold, or even longer, for FIX. The implications in clinical practice are discussed, highlighting the potential role of these products in either improving convenience and adherence through prolongation of the interval between infusions or increasing trough levels and the safety against bleeds maintaining current intervals. ${ }^{15}$ While awaiting further information about the "real-life" use of EHL products, exciting perspectives also come from alternative therapeutic approaches not based on the replacement of the missing factor. Ongoing research aims to produce therapeutic agents with reduced immunogenicity and yet equally effective in patients with or without inhibitors. These new classes of hemostatic agents act mainly by bypassing the need for FVIII and FIX in tenase formation (recombinant activated factor VII fusion protein with albumin, the bispecific antibody against factor $\mathrm{X}$, and activated FIX ACE910), quenching anticoagulant pathways (the antitissue factor pathway inhibitor concizumab, the short-interfering RNA ALN-AT3 suppressing synthesis of antithrombin) and enhancing the activity of some coagulation factors (Factor $\mathrm{V}$ or X variants) or stabilizing the fibrin clot (Factor XIII). Current knowledge on the development of these novel molecules is reviewed by Mannucci and colleagues. ${ }^{16}$ Further interest for these products are related to the potential earlier and easier prophylaxis implementation thanks to subcutaneous administration and prolonged half-life, and a low immunogenicity with possible prevention of inhibitor development in high-risk patients. ${ }^{16}$

In the interim, prophylaxis regimens with standard factor concentrates remain the treatment of choice for patients with hemophilia, particularly in children. ${ }^{2,18}$ However, a crucial barrier to widespread use of long-term prophylaxis is the remarkably high cost of factor concentrates. Indeed, economic constraints make the usually adopted high- or intermediate-dose prophylaxis regimens unaffordable in most countries. Windyga addresses this issue, reviewing the increasing literature data suggesting that low-dose prophylaxis regimens offer significant benefits over on-demand treatment with substantially comparable factor concentrate consumption. These approaches result in cost-saving compared with higher dose regimens and are useful for implementing prophylaxis in low-income countries. ${ }^{19}$ However, the actual cost-effectiveness of adopting different prophylaxis regimens, or personalized approaches with both standard and EHL products, should consider long-term outcomes, primarily the impact on patients' joint status. In this respect, as regards 
imaging techniques, ultrasound (US) is increasingly recognized as an easily available and helpful tool to monitor hemophilic arthropathy. Di Minno and colleagues summarize the literature data on the evaluation by joint US of markers of disease activity (joint effusion and synovial hypertrophy) and of degenerative damages (osteochondral changes) and the scoring systems proposed for staging. ${ }^{20}$ They show that US is the fastest and most reliable technique to detect acute conditions, such as hemarthrosis, and joint involvement since early signs, monitoring synovial hypertrophy and osteochondral changes. US data may improve information from clinical examination and be helpful for enabling treatment decisions on a personalized basis and in identifying joints needing careful follow-up and second-level imaging such as magnetic resonance imaging. ${ }^{20}$

Four subsequent chapters in this issue concern challenges always faced in replacement treatment of CBD, namely pathogen safety, thrombotic complications, and inhibitor development. The latter, in particular, is currently the most serious complication of therapy in hemophilia A, due to its incidence in PUPs and the impact in terms of patients' morbidity and impairment of quality of life and of increase in healthcare costs. $^{2}$ In the frame of the multifactorial pathogenesis involving many factors, both genetic (unmodifiable) and environmental (including treatment, potentially modifiable), Batorova and coworkers add their contribution to the never-ending debate regarding the role in inhibitor development of the type of FVIII concentrate. ${ }^{21}$ In a prospective analysis of all 61 PUPs born and treated in Slovakia since the introduction of safe FVIII concentrates in 1990, they report a significantly higher inhibitor risk in PUPs receiving recombinant products versus those treated with plasma-derived ones. Moreover, most inhibitor patients were tolerized after immune tolerance induction with plasma-derived FVIII concentrates. These intriguing results, however, should be interpreted cautiously, in particular due to the small study population with few patients on recombinant products. ${ }^{21}$ In previously treated patients (PTPs), the incidence of inhibitors is much lower and, even in this setting, the role of treatmentrelated factors is still debated, with many patients and physicians concerned about risk in switching FVIII products. Product changes may occur because of the development of new concentrates considered safer, as well fewer side effects, convenience issues, or economic reasons. From a literature review addressing the safety of switching FVIII concentrates, Coppola and colleagues did not find evidence of increased inhibitor risk in studies either in patients during the first 50 to 75 exposure days or in PTPs, from registration and postmarketing studies of new FVIII concentrates and, in particular, from studies reporting data of national cohorts of patients switching products. ${ }^{22}$ Caution should be adopted in some groups of patients with higher inhibitor risk; however, perceived more than evidence-based challenges, in the presence of clinical needs, should not lead to missing the advantages of switching FVIII concentrates, in particular in this era of newer products with improved properties recently introduced or available in few years. ${ }^{22}$ Thrombotic complications are relatively uncommon in patients with $\mathrm{CBD}$, although these may be expected to increase as a natural outcome of increased life expectancy. ${ }^{23}$ In some clinical settings, however, the risk may be higher due to the characteristics of the replacement products or to the pathophysiological background. This is the case, for example, of the patient with afibrinogenemia reported by Santoro and coworkers; this patient developed a massive arterial thrombosis of left lower limb, requiring a complex management balancing multiple antithrombotic pharmacological and endovascular strategies with prophylaxis of bleeding. ${ }^{24}$ The authors also performed a literature search, reviewing similar thrombotic complications and addressing the unsolved question of the relationship of thrombosis in this setting with replacement treatment. ${ }^{24}$ Finally, the review by Farrugia points out the importance of maintaining a high level of consciousness regarding pathogen safety of blood products by the community of patients, treaters, and healthcare policy makers. ${ }^{25}$ Indeed, although the stringent measures which ensure the safety of plasmaderived factor concentrates are very effective, pathogen transmission by other blood-derived therapeutics has continued due to a lack of effective technology and the emergence of new agents which transmit disease. Therefore, the continued role for these products in CBD care, particularly in emerging countries, challenges to maintain blood safety measures, these issues being more current than historical. ${ }^{25}$

The last chapter in this issue by Tagliaferri and colleagues reports a successful model for addressing the universal challenge of the emergency care of CBD patients, given the lack of specialists readily available. ${ }^{26}$ Thanks to a web-based platform collecting patients' records and providing bleedingand disease-specific algorithms for treatment, a network joining all the emergency departments and the hemophilia treatment centers in the Emilia-Romagna region (Italy) was activated. After an intensive training of personnel, the first 2year results of this project are encouraging, showing improvements in the management of patients, particularly in terms of reduced triage-assessment and triage-treatment times and increased medical advice from the hemophilia centers. $^{26}$

In conclusion, while thanking all the authors for these excellent contributions, we really think that the chapters presented in this issue of Seminars in Thrombosis and Hemostasis provide the readers with an effective and interesting overview of the current scenario of CBD, increasingly oriented, like other medical fields, to personalized approaches and "precision medicine."27 A series of challenges and open issues remain to be faced; however, patients and treaters can actually foresee exciting perspectives in the near future.

\section{References}

1 Franchini M, Favaloro EJ, Lippi G. Newer hemostatic agents. Semin Thromb Hemost 2015;41(7):802-808

2 Rocino A, Coppola A, Franchini M, et al; Italian Association of Haemophilia Centres (AICE) Working Party. Principles of treatment and update of recommendations for the management of haemophilia and congenital bleeding disorders in Italy. Blood Transfus 2014;12(4):575-598 
3 Gouw SC, van der Bom JG, Ljung R, et al; PedNet and RODIN Study Group. Factor VIII products and inhibitor development in severe hemophilia A. N Engl J Med 2013;368(3):231-239

4 Eckhardt CL, van Velzen AS, Peters M, et al; INSIGHT Study Group. Factor VIII gene (F8) mutation and risk of inhibitor development in nonsevere hemophilia A. Blood 2013;122(11):1954-1962

5 Peyvandi F, Di Michele D, Bolton-Maggs PH, Lee CA, Tripodi A, Srivastava A; Project on Consensus Definitions in Rare Bleeding Disorders of the Factor VIII/Factor IX Scientific and Standardisation Committee of the International Society on Thrombosis and Haemostasis. Classification of rare bleeding disorders (RBDs) based on the association between coagulant factor activity and clinical bleeding severity. J Thromb Haemost 2012;10(9): 1938-1943

6 Holm E, Abshire TC, Bowen J, et al. Changes in bleeding patterns in von Willebrand disease after institution of long-term replacement therapy: results from the von Willebrand Disease Prophylaxis Network. Blood Coagul Fibrinolysis 2015;26(4):383-388

7 Tosetto A. Bleeding assessment tools: limits and advantages for the diagnosis and prognosis of inherited bleeding disorders. Semin Thromb Hemost 2016;42(5):463-470

8 Lippi G, Franchini M, Favaloro EJ. Diagnostics of inherited bleeding disorders of secondary hemostasis: an easy guide for routine clinical laboratories. Semin Thromb Hemost 2016;42(5):471-477

9 Favaloro EJ. Diagnosing von Willebrand disease: a short history of laboratory milestones and innovations, plus current status, challenges, and solutions. Semin Thromb Hemost 2014;40(5): 551-570

10 Castaman G, Federici AB. Type 2B von Willebrand disease: a matter of plasma plus platelet abnormality. Semin Thromb Hemost 2016; 42(5):478-482

11 Favaloro EJ, Pasalic P, Curnow J. Type 2M and type 2A von Willebrand disease: similar but different. Semin Thromb Hemost 2016;42(5):483-497

12 Saccullo G, Makris M. Prophylaxis in VWD: coming of age? Semin Thromb Hemost 2016;42(5):498-506

13 Castaman G, Eckardt C, van Velzen A, Linari S, Fijnvandraat K. Emerging issues in diagnosis, biology and inhibitor risk in mild hemophilia A. Semin Thromb Hemost 2016;42(5):507-512

14 Giangrande P. The future of hemophilia treatment: longer-acting factor concentrates versus gene therapy. Semin Thromb Hemost 2016;42(5):513-517
15 Berntorp E, Andersson NG. Prophylaxis for hemophilia in the era of long-acting FVIII/FIX products. Semin Thromb Hemost 2016; 42(5):518-525

16 Mannucci PM, Mancuso ME, Santagostino E, Franchini M. Innovative pharmacological therapies for the hemophilias not based on deficient factor replacement. Semin Thromb Hemost 2016;42(5): 526-532

17 Nathwani AC, Reiss UM, Tuddenham EG, et al. Long-term safety and efficacy of factor IX gene therapy in hemophilia B. N Engl J Med 2014;371(21):1994-2004

18 Coppola A, Tagliaferri A, Di Capua M, Franchini M. Prophylaxis in children with hemophilia: evidence-based achievements, old and new challenges. Semin Thromb Hemost 2012;38(1):79-94

19 Windyga J. Is continuous low-dose prophylaxis superior to ondemand treatment for patients with haemophilia? Semin Thromb Hemost 2016;42(5):533-540

20 Di Minno MND, Ambrosino P, Quintavalle G, et al. Assessment of joint status by ultrasound in hemophilia: where do we stand? Semin Thromb Hemost 2016;42(5):541-549

21 Batorova A, Jankovicova D, Morongova A, et al. Inhibitors in severe hemophilia A: 25-year experience in Slovakia. Semin Thromb Hemost 2016;42(5):550-562

22 Coppola A, Marrone E, Conca P, et al. Safety of switching factor VIII products in the era of evolving concentrates: myths and facts. Semin Thromb Hemost 2016;42(5):563-576

23 Coppola A, Santoro C, Franchini M, et al. Emerging issues on comprehensive hemophilia care: preventing, identifying, and monitoring age-related comorbidities. Semin Thromb Hemost 2013;39(7):794-802

24 Santoro C, Massaro F, Venosi S, et al. Severe thrombotic complications in congenital afibrinogenemia: a physiopathologic and management dilemma. Semin Thromb Hemost 2016;42(5): 577-582

25 Farrugia A. Safety issues of plasma-derived products for treatment of inherited bleeding disorders. Semin Thromb Hemost 2016; 42(5):583-588

26 Tagliaferri A, Di Perna C, Biasoli C, et al. A Web site to improve the management of patients with inherited bleeding disorders in the emergency department: results at 2 years. Semin Thromb Hemost 2016;42(5):589-598

27 Collins FS, Varmus H. A new initiative on precision medicine. N Engl J Med 2015;372(9):793-795 